\title{
AN ADAPTIVE COMPUTATIONAL MODEL FOR THRESHOLD BASED VM MIGRATION AND JOB SCHEDULING IN CLOUD
}

\author{
Anitha $\mathrm{R}^{1}$ and $\mathrm{C}$ Vidyaraj ${ }^{2}$ \\ ${ }^{1,2}$ The National Institute of Engineering, Mysuru, India \\ ${ }^{1}$ anithamahendra123@gmail.com, ${ }^{2}$ vidya_rajc@yahoo.com
}

Abstract - Cloud computing is considered as a most promising technique for offering the strong significant resources for computation for huge data by taking the advantage of virtual machine configurations where multiple operating systems are configured where multiple application applications are deployed to perform the several tasks. However, during peak time, huge number of requests are processed through the virtual machines where overloading phase of virtual machine may occur which may delay the task completion process resulting in degraded performance of cloud computing system. In order to deal with these issue, virtual machine migration strategy is introduced where overloaded virtual machines are migrated to perform the task in optimal time duration which can help to finish the task on the pre-assigned time duration and can save the energy consumption. During last decade, significant amount of work has been carried out in this field of virtual migration but achieving desired performance is still challenging. In order to deal with this issue, here we present a novel approach where we considered resource availability related information for VM allocation. In the next phase, threshold-based migration scheme is implemented based on the computing resources. Finally, an experimental study is presented for VM migration using proposed technique and a comparative study is also presented which shows that proposed approach achieves better performance when compared with the state-of-art techniques of VM migration.

Keywords - VM Migration, Scheduling, Resource Utilization, Load Balancing, Cloud

\section{INTRODUCTION}

During last two decades, the web-based communication system such as Internet and WWW have noticed an expeditious growth which steered towards the huge amount of information available online. Additionally, use of the various application such as scientific, social and engineering also increased which generates the huge amount of data in structured and unstructured terms which need to be analyzed, processed and stored [1]. Due to increasing size of data, an efficient modeling is required for computing the processing these data hence techniques for data processing and storage have gained attraction from the researchers and industries. The technical growth of these systems has led towards the successful and cheaper implementation of Internet and computing resources. In order to develop an efficient model for analyzing, processing and storing these huge data, a new schematic model is presented known as Cloud Computing.

This technique of Cloud computing is considered as prevalent and compelling for information technology (IT) field in various terms. This computing model is Internet

Received: January 29, 2019

Reviewed: May 28, 2019

Accepted: June 2, 2019

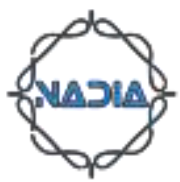


based development and also it uses computer technology. This model includes three main components which formulates complete cloud computing architecture which are known as platform as a service (PaaS), infrastructure as service (IaaS) and software as a service $(\mathrm{SaaS})$ [2]. Cloud computing has significant nature of computation and provides the scalable architecture which helps to develop a virtualized model where any job or query can be executed in a virtual manner. Virtualization is a process which allows the computing servers to assume multiple operating systems where multiple applications and tasks can be performed on various systems. Moreover, this technique provides the online migration strategy where any overloaded server can be migrated to other server which is having less overhead, resulting in efficient task completion. In order to satisfy the task completion criteria of client, cloud providers urge to increase the number of data center for computation. Generally, data centers are complicated but capable to facilitate the better resource utilization and maximizing the cloud performance [3].

According to conventional data center, physical servers which are responsible for performing under higher workloads, are assigned for any specific application. However, these systems suffer from various aspects such as expensive, require higher maintaining cost, space, resource utilization and energy wastage etc. which degrade the computing performance. These issues can be addressed using virtualization technique which enables the consolidation of server and improve the resource utilization. However, different type of workloads and assignments are given to the server which becomes unpredictable causing the resource imbalance resulting in deteriorated performance. Various aspects are present which make cloud data center dynamic and unpredictable such as resource utilization fluctuation, data arrival rate, irregular data patterns and query assigned by the consumer etc. These issues can cause load unbalancing in the cloud data centers which may lead to the degraded performance and SLA (Service Level Agreement) violations. These issues can be addressed using load-balancing schemes where dynamic queries are distributed over other servers to balance the workloads. This technique of load-balancing, satisfies the resource utilization criteria and improves the performance of overall system. A significant process of load balancing can help to achieve the optimal performance of resource utilization, scalable and reduces the response time for given query. Sufficient amount works have been carried out in this field of load balancing for cloud computing applications [4-5].

As discussed earlier, virtualization is considered as a promising technique in this field which can enhance the computation performance of cloud computing systems. Due to increasing demand of cloud based application, more number of data centers are required to perform the various task and assure the on-time task completion process. hence, this can be achieved using virtualization of servers known as virtual machine. Main aim of virtual machine is to achieve desired results for resource utilization and task completion for each client application [6]. Cloud-based resources are highly expensive to implement; thus, performance optimization and server consolidation are some essential tasks which need to be performed for any task/query. For server consolidation process, VM (virtual machine) placement is an important task. Various studies have been presented which shows that the traffic variability is increasing due to adoption of various enterprises and this variable traffic effects the performance of cloud systems. To cater this issue of traffic variability, scheduling based techniques shows significant impact on the performance but scheduling based techniques fail to perform for dynamic traffic demands and overhead reduction [7]. Researchers have concluded that this issue of handling dynamic traffic can be addressed by using virtual machine migration schemes [8] and local resizing [9]. According to local resizing process, VM configuration changes adaptively whereas live migration provides VM placement for servers which are ideal for a certain period. Moreover, live migration is 
an important task in this field which provides efficient data delivery. In [10], authors presented a technique for virtual machine migration with the help of pre-copy algorithm. Pre-copy and post-copy algorithms are widely used for migration of VMs but further performance improvement is also needed in these approaches. Hence, a hybrid scheme is developed which combines both pre-copy and post copy algorithm for migration of VMs. During this process of migration, load balancing is also an important parameter. Tiwari et al. [11] introduced a combined scheme of load balancing and virtual machine migration. Furthermore, these techniques of VM migration can be categorized such as energy efficient migration, load balancing migration and fault tolerant migration. Several studies have been presented for VM migration for improving the performance of cloud systems. Conventional techniques suffer from various issues such as higher resource utilization, downtime and communication overhead hence there is a need to develop an efficient technique for virtual machine migration which can improve the performance.

In this work, our main aim is to develop an adaptive approach for virtual machine migration which can improve the overall performance by reducing the overhead parameters. The main contributions of this work are as follows:

(a) First of all, we present a general introduction about cloud computing, virtualization \& its advantage

(b) We discuss about recent techniques and trends for virtual machine migration

(c) An adaptive computation model is presented for improving the virtualization and migration.

(d) A comparative experimental study is also presented to show the robust performance of proposed approach for cloud computing.

Remaining article is arranged as: section II presents recent models and studies of cloud computing, proposed study is described in section III, section IV presents the experimental studies and comparison and finally, concluding remarks about proposed model are given in section $\mathrm{V}$.

\section{LITERATURE SURVEY}

A brief discussion about recent techniques for load balancing, virtualization and VM migration is presented in this section. Cloud computing has been considered as an attractive area of research to provide efficient computing and storage space for clients. In order to perform these type of tasks, cloud providers need to establish multiple data centers. These data centers are responsible to perform the assigned tasks. During computation process, huge amount of energy is required which cases more power consumption [12]. Similarly, for better performance of cloud systems and satisfaction of clients, assigned task must be finished in a given time duration. Due to increasing demand of cloud computing based system, multiple number of tasks are assigned which causes overhead and leads to the degrade performance. To overcome this issue, load balancing is considered as most promising scheme [13]. However, energy consumption still remains a challenging research area in this field. Recently, several techniques have been presented to overcome the issue of power consumption which include migration schemes and without migration schemes. In this process, live $\mathrm{VM}$ migration is considered as a significant technique for reducing the energy consumption [14]. According to VM migration process, a running VM is transferred to another active node if it is overloaded or down. This process shows significant impact for energy saving in cloud computing systems. Generally, VM migration schemes are not as simple because it includes other stages such as VM placement [15], VM consolidation [16] etc. 
Khosravi et al., [17] discussed about energy consumption issues in cloud data centers and estimated that how much energy can be preserved using renewable energy for data centers. Generally, renewable energy has irregular nature of production which may cause issues for power supply and generation. Hence, virtual machine migration near the datacenter can help to achieve the desired performance. In this work, authors presented an offline algorithm where renewable level of the energy sources is already known.

Duggan et al., [18] focused on the live VM migration for cloud computing systems and also discussed that increased consumption of network resources may lead to the longer time requirement for VM migration. Currently, various industries use manual process for VM migration which is addressed in this work and network aware live VM migration scheme is presented which considers the current demand of the network resources and performs the suitable action. In order to learn the requirement parameters, artificial intelligence based reinforcement learning scheme is introduced which learns the network demand patterns and optimal scheduling time for any given network traffic model. Wu et al., [19] also focused on the live VM migration and discussed about its importance for cloud computing systems. Conventional techniques of migration are based on the pre-copy and post-copy methods of migration which require more time hence time-series based pre-copy and post-copy based methods presented here. General model of pre-copy techniques can provide the better migration by reducing the time taken for migration but it may lead to longer downtime which will cause more time for task completion. The complete process of migration is categorized into three main phases as (a) Push phase (b) stop and copy and (c) pull phase and the performance of migration is measured by computing downtime and migration time. In this approach, a memory prediction scheme is also presented which can select the pages to migration during pre-copy or stop and copy phase.

Recently, private clouds are also widely adopted by various industries. Private clouds have very limited resources hence a proper load lancing, minimal energy consumption, resource utilization is required to obtain the optimal performance from the computing architecture. Based on these assumption, Sohrabi et al., [20] presented a novel approach of VM migration for private cloud computing systems. A novel combined approach is presented in this work where selection of VMs and VM mapping are discussed together for energy enhancement. Due to unpredictable nature of incoming task, it becomes very crucial tsk to optimize the performance. Hence, a new approach with adaptive computation process is introduced where Bayesian interference is used for likelihood estimation for VM migration.

As discussed before, for VM migration consolidation and placement play important roles. Various methodologies have been presented for VM placement and consolidation to enhance the system performance. Recent growth in efficient computation of cloud computing, cloud providers such as Microsoft Azure and Amazon have come up with IaaS based new cloud model where MapReduce based schemes are utilized to perform the task for huge workloads. It is considered that the IaaS have multiple pricing option, we discuss about batch-oriented consolidation and placement of virtual machines. In this work, DVFS based TRP-FS scheme is introduced for task management and VM consolidation along with this it also guarantees to follow the SLA criteria [21].

Zola et al., [22] also focused on the cloud performance optimization using datacenter virtualization and VM migration process. in order to reduce the energy consumption, cloud operators consolidate various virtual machine to less number of physical servers which may lead to the SLA violations during peak load hence a proper method is required for VM migration. Therefore, a novel approach presented using optimization computation process. in this process, VM consolidation is modeled in the robust Mixed Integer Linear Program which also considers the resource-based constraints of cloud. Abdullah et al., [23] presented a heuristic approach for energy consumption minimization and satisfying the SLA criteria. In order to allocate the VMs, fast best-fit decreasing (FBFD) algorithm is presented and 
dynamic utilization rate (DUR) algorithm is also introduced for space utilization and VM migration.

\section{PROPOSED MODEL}

\subsection{PROBLEM FORMULATION}

In this section, a complete discussion about proposed approach for VM migration is presented. First of all, we present a problem formulation model where we discuss about virtual machine, physical machine and overloaded VMs to formulate the optimization problem. Let us consider that a set of virtual machines $V$ is presented as $V M=$ $\left\{V M_{1}, V M_{2}, V M_{3} \ldots, V M_{n}\right\}$, set of physical machines is given as $P M=$ $\left\{P M_{1}, P M_{2}, P M_{3} \ldots, P M_{n}\right\}$ whereas set of total overloaded VMs is given as $O=$ $\left\{V M_{1}, V M_{2}, V M_{3} \ldots, V M_{k}\right\}$ where $O \subset V M$. In this work, a data center network is presented in a dependency graph as $G=(V, E)$ where vertices and edges of network are denoted by $V$ and $E$ respectively where set of edges can be defined as $E=$ $\left(V M_{i}, V M_{j}\right)$. Similarly, traffic demand also considered for each edge of the network, represented as $W\left(V M_{i}, V M_{j}\right)$ which has relationship with network vertices as $W\left(V M_{i}, V M_{j}\right) \propto\left(V M_{i}, V M_{j}\right)$ i.e. traffic between $V M_{i}$ is proportional to the traffic transferred to $V M_{j}$. Further, we define load on the network in the form of vector which contains various requirements of virtual machine such as CPU configuration, memory and load. Another parameter is defined known as capacity of the server. During migration process, time, energy and resource consumption are considered as main parameters. According to migration process, a virtual machine $V M_{i}, V M_{j}$ is migrated to the corresponding physical machine then the cost of migration can be given as:

$$
C\left(V M_{i}, P M_{k}, V M_{j}, P M_{l}\right)=\operatorname{Dist}\left(P M_{k}, P M_{l}\right) \times W\left(V M_{i}, V M_{j}\right)
$$

Where $C$ denotes cost, Dist is given for distance computation function

$\operatorname{Dist}\left(P M_{k}, P M_{l}\right)$ is estimated based on the latency, total number of hop and delay parameters between considered physical machines $P_{k}$ and $P_{l}$. Based on these discussions, we define

$$
X_{i k}=\left\{\begin{array}{c}
1 \text { if } V M_{i} \text { is assgned to the physical machine } P M_{1} \\
0 \text { otherwise }
\end{array}\right\}
$$

According to this assumption, if any virtual machine $V M_{i}$ is placed on the physical machine $P M_{k}$ then $X_{i k}$ is assigned as 1 otherwise it is assigned to 0 . Based on these models, an optimization framework can be developed as:

$$
\text { minimze } \sum_{\alpha} C\left(V M_{i}, P M_{k}, V M_{j}, P M_{l}\right) \times X_{i k}^{j l}
$$

In this optimization $\alpha$ is given as $\alpha=\{(i, j, k, l)|i<j, k<l, j<| V M|, l<| P M \mid\}$. This helps to minimize the overhead on the VMs during migration process by placing the virtual machines near the datacenter. Furthermore, we examine the VM overloaded criteria, as:

$$
\sum_{k}^{|P M|} X_{i k}=1, \forall_{i}, V M_{i} \in O
$$

This helps us to identify the overloaded virtual machine and to avoid this, a virtual machine $V M_{i}$ must be placed at the physical machine $P_{k}$. To establish the relation between the load and physical machine capacity whether the load on the machine is equal to the capacity, can be obtained by: 


$$
\sum_{k}^{|V M|} X_{i k} \times \text { Load }_{i} \leq \text { capacity }_{k}, \forall_{k}, P M_{k} \in P M
$$

Finally, the overloaded VMs can be resolved by using $X_{i k}=x_{i k}, x_{i k}$ denotes the non-overloaded VMs.

\subsection{MIGRATION METHODOLOGY}

Previous sub-section describes the problem formulation for VM migration in cloud computing where an optimization problem is formulated. In this section, we describe the proposed approach to solve the optimization problem. According to the proposed approach, we assume that task request is provided by any data-intensive application, moreover bandwidth and storage parameters of VMs are already known.

In this context of cloud computing, any input data can be distributed into multiple blocks or parts which can be stored with a certain physical distance. This data distribution matrix is given as $D_{n \times m}$ which can be computed as:

$$
D_{n \times m}=\left|\begin{array}{cccc}
d_{1,1} & d_{1,2} & \ldots & d_{1, m} \\
d_{2,1} & d_{2,2} & \ldots & d_{2, m} \\
\ldots & \ldots & \ldots & \ldots \\
d_{n, 1} & d_{n, 2} & \ldots & d_{n, m}
\end{array}\right|
$$

In this method, $m$ denotes the column number which represents the total amount of data to be processed and number of rows denotes the total number of storage nodes. Furthermore, for each member of data i.e. column of data matrix, it should follow the relationship as DataSize $m=\sum_{i=1}^{n} d_{i, m}$ and the speed between VM and data storage is given as $S=\operatorname{Speed}(s, \Delta t)$ where $s$ denotes the packet size and $\Delta t$ denotes the time taken for packet transfer. In the cloud computing systems, virtual machines are hosted on the physical machines and the status of VMs is denoted as $\delta_{i, j}$ where $i$ denotes the number of nodes available to store the data in a matrix $\mathcal{D}_{n, m}$.

Let us consider that upcoming task expecting to access $d_{1}$ at this stage it is checked that the computation resources are satisfying the task completion requirements. Based on this, the computing resource sets can be given as:

$$
C R=\{P M, M\}
$$

Where $P M$ denotes the capacity of processor on the physical machine and $M$ denotes the memory capacity. The current status of occupied resources and memory can be expressed as:

$$
C R_{\text {busy }}=\left\{P M_{V M, i}, M_{V M, i}\right\}
$$

Hence, available computing resources can be given as

$$
C R_{\text {available }}=\left\{P M_{\text {available }}, M_{\text {available }}\right\}=\left\{P M-\sum_{i=1}^{n} P M_{V M}, M-\sum_{i=1}^{n} M_{V M}\right\}
$$

This complete process of network resource availability modeling is given in the algorithm 1 . 
Input: Network status, data storage information, upcoming application request

Step 1: compute data transfer time as $T=\mathcal{S}_{i, j} \cdot D_{n, m}$

Step 2: process through the complete time matrix and obtain parameter as minimum $\sum_{y=1}^{\omega} T_{x, y} \& \& C R_{\text {avaialble }}>C R_{V M_{\text {arriving }}}$

Step 3: return value of $x$

Step 4: allocate arriving VMs on the physical machine

Step 5: if VM allocation is successful

Step 6: $C R_{\text {avialble }}=\left\{P M_{\text {available }}-P M_{V M_{\text {arriving }}}, M_{\text {available }}-P M_{V M_{\text {arriving }}}\right\}$

Step 7: end

In previous discussion we have studied that the nature of cloud computing system is unpredictable hence network latency may occur resulting in degraded performance for data intensive applications. This latency issue may cause SLA violations which are not allowed by the cloud providers hence a proper migration is required which can also satisfy the SLA standard along with efficient computation.

Generally, a threshold is provided for any SLA based application cloud computing. If the time duration is exceeding from the pre-defined SLA threshold. Let us consider any application which will interact with the data $d$ then the total time requirement can be denoted as $\sum_{y=1}^{\omega} T_{x, y}$ and it is assumed that the $T_{S L A}$ is a threshold time which is requested by the user for specific application. Based on this, thee migration scheme can take place if $\sum_{y=1}^{\omega} T_{x, y}>T_{S L A}$. This process is given in algorithm 2 .

Input: Requested threshold, time matrix for data transfer, upcoming application request

Step $\quad 1:$ if $\sum_{y=1}^{\omega} T_{x, y}>T_{S L A}$

Step 2: process through the complete time matrix for data transfer

Step 3: find minimum $\sum_{y=1}^{\omega} T_{x, y} \& \& C R_{\text {avaialble }}>C R_{V M_{\text {arriving }}}$

Step 4: return $x$

Step 5: reallocate the VMs to the physical machine

Step 6: end if

Step 7: if VM allocation is successful

Step 8: $C R_{\text {avialble }}=\left\{P M_{\text {available }}-P M_{V M_{\text {arriving }}}, M_{\text {available }}-P M_{V M_{\text {arriving }}}\right\}$

Step 9: end

\section{EXPERIMENTAL STUDY}

This section provides experimental analysis of proposed approach of VM migration. In this study, we have evaluated the performance based on varied number of virtual machines, node utilization threshold and number of iterations. We have considered up to $200 \mathrm{VMs}$ where total 40 runs are performed for performance measurement. Energy consumption performance is also computed by using varied number of node utilization 
threshold. Figure 1 shows a comparative performance based on number of VMs and number of hosts.

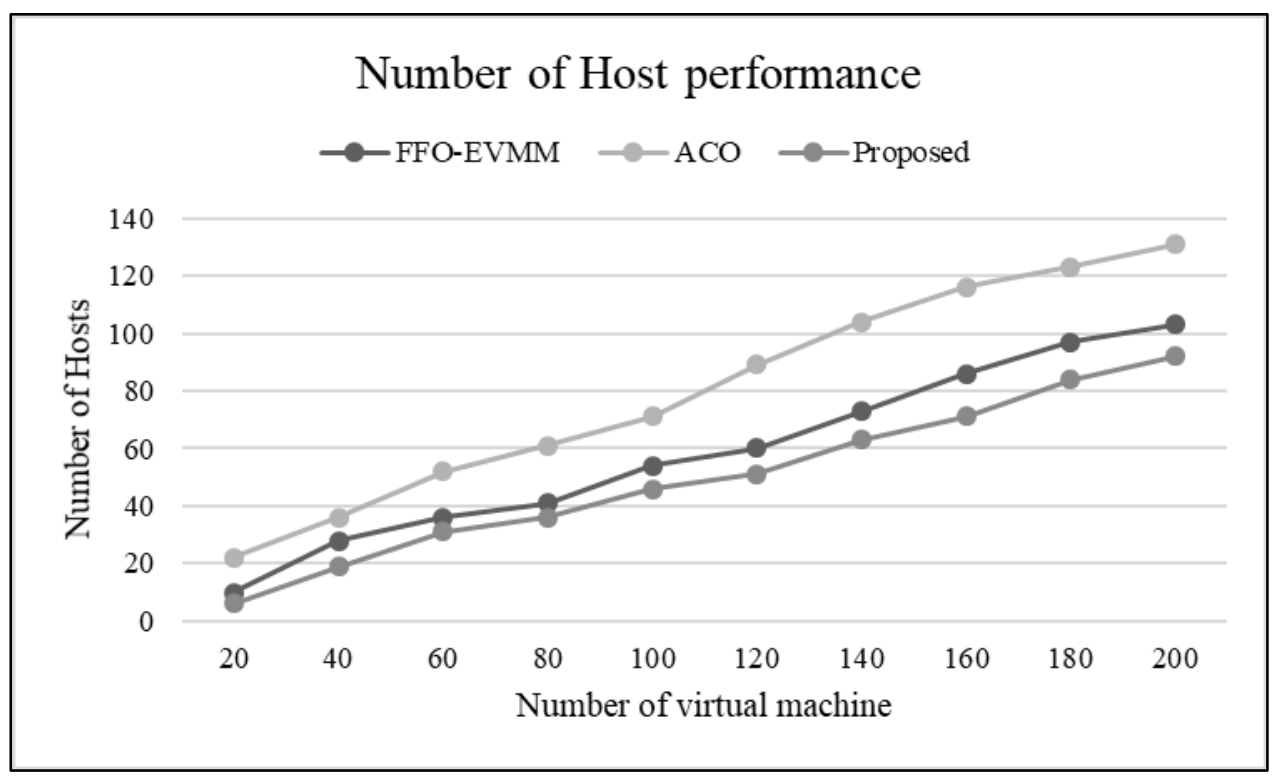

Fig. 1 Number of Hosts Performance

Above given Figure 1 shows a comparative performance for varied number of virtual machines where total $200 \mathrm{VMs}$ are considered and required number of hosts are computed. Proposed approach uses less number of hosts when compared with existing approaches [24]. According to this experiment, it is obtained that total average number of required hosts are $80.5,58.8$ and 49.9 for ACO, FFO-EVM and proposed approach respectively.

Similarly, we computed total number of VM migration during a computation process and compared with existing schemes. This experimental performance is depicted in Figure 2.

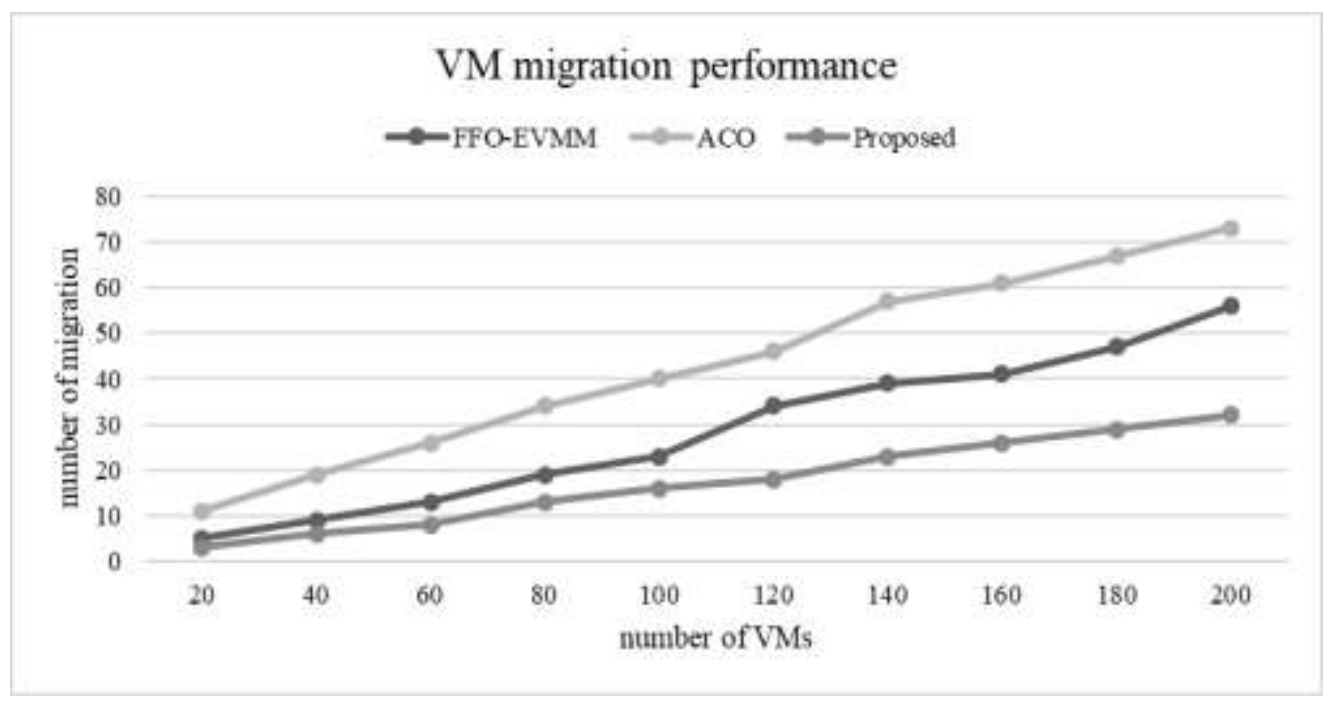

Fig. 2 VM Migration Performance

The experiment presented in Figure 2 shows the comparative performance of VM migration during computation phase. Experimental study shows that the proposed 
approach requires less number of migration hence it can provide on time task completion. In this experiment, we show a comparative performance for varied number of VMs and evaluated the performance in terms of total number of migration. The obtained total number of average migration are obtained as 28.6, 43.4 and 17.4 for FFO-EVM, ACO and proposed approach. The proposed approach requires less number of migration which provide the robustness to the system for task completion.

In next phase, we present a comparative performance measurement in terms of average energy consumption for varied node utilization threshold.

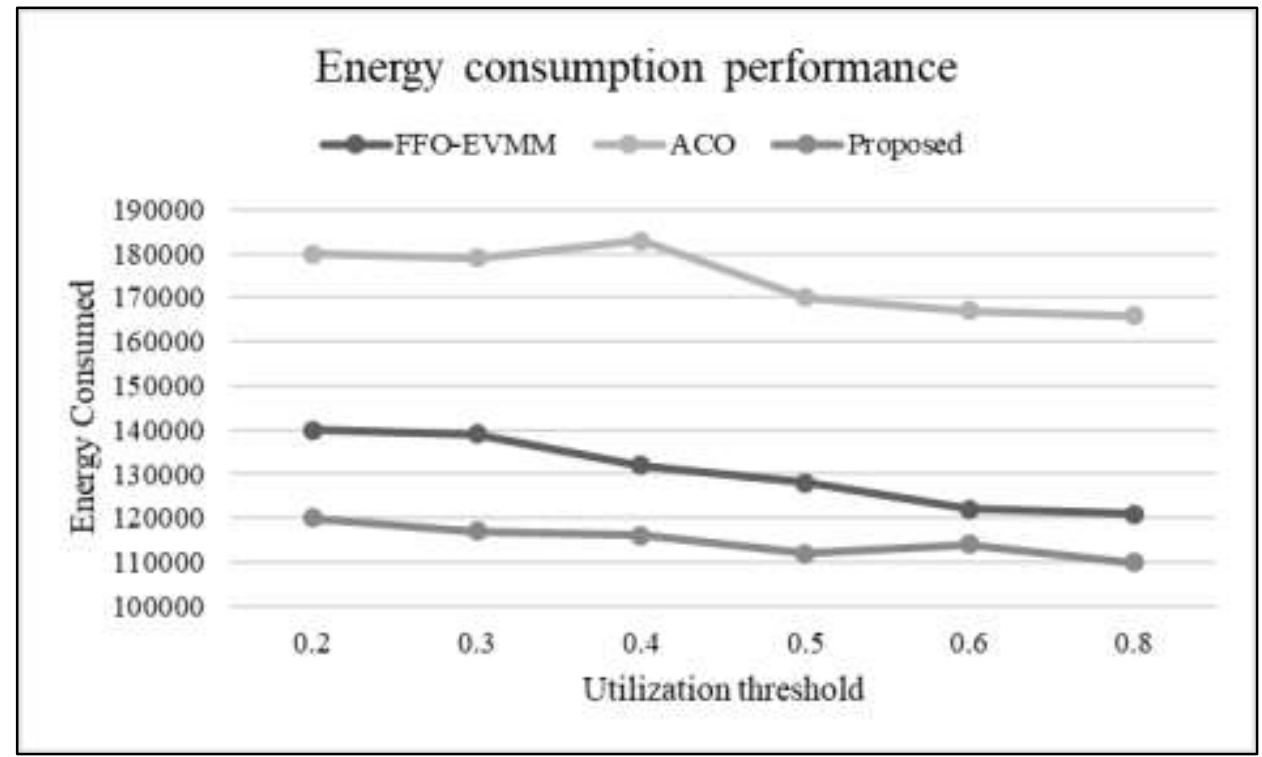

Fig. 3 Energy Consumption Performance

Figure 3 shows a comparative study in terms of energy consumption and compared the performance of proposed approach with the state-of-art techniques such as FFOEVM and ACO. Performance comparison shows that proposed approach obtains better performance.

\section{CONCLUSION}

This article mainly deals with the development of adaptive and robust approach implementation for virtual machine migration. According to the cloud computing, initially virtual machine configurations are placed on the varied locations in the form of data centers. These virtual machines are equipped with operating systems and applications which can perform the desired task but as the data traffic increases, inappropriate VM allocation may lead towards the VM overloading resulting in performance degrade of the system. In this work, we addressed this issue of virtual machine migration and presented a novel approach. According to the proposed approach, first of all we formulate a VM migration problem where multiple VMs, physical machines considered along with the overloaded VMs. The problem is constructed as optimization problem whose main aim is to minimize the load over a VMs. In the next phase of the work, we focus on the VM allocation task by considering several other parameters such as Network status, data storage information, upcoming application requests. After performing the successful allocation, we aim on the SLAs based on the threshold and resource availability. An extensive experimental study is carried out and compared with the existing techniques of VM migration. Experimental analysis shows that proposed approach achieves better performance when compared with existing models. 


\section{REFERENCES}

[1] Sadooghi, Iman, et al. "Understanding the performance and potential of cloud computing for scientific applications." IEEE Transactions on Cloud Computing 5.2 (2017): 358-371.

[2] Rastogi, Garima, et al. "Deployment of Cloud Using Open-Source Virtualization: Study of VM Migration Methods and Benefits." Big Data Analytics. Springer, Singapore, 2018. 553-563.

[3] Rodrigues, Tiago Gama, et al. "Hybrid Method for Minimizing Service Delay in Edge Cloud Computing Through VM Migration and Transmission Power Control." IEEE Transactions on Computers 66.5 (2017): 810-819.

[4] Mousavi, Seyedmajid, Amir Mosavi, and Annamária R. Varkonyi-Koczy. "A load balancing algorithm for resource allocation in cloud computing." International Conference on Global Research and Education. Springer, Cham, 2017.

[5] Ghomi, Einollah Jafarnejad, Amir Masoud Rahmani, and Nooruldeen Nasih Qader. "Loadbalancing Algorithms in Cloud Computing: A Survey." Journal of Network and Computer Applications (2017).

[6] Tsai, Linjiun, and Wanjiun Liao. Virtualized Cloud Data Center Networks: Issues in Resource Management. Springer, 2016.

[7] N. L. S. da Fonseca, R. Boutaba, -Cloud Services, Networking, and Managementll, John Wiley \& Sons, 2015.

[8] Deshpande, Umesh, and Kate Keahey. "Traffic-sensitive live migration of virtual machines." Future Generation Computer Systems 72 (2017): 118-128.

[9] S. Fiore, G. Aloisio, - Grid and Cloud Database Managementll, Springer Science \& Business Media, 2011.

[10] atel, Minal, Sanjay Chaudhary, and Sanjay Garg. "Performance Modeling and Optimization of Live Migration of Virtual Machines in Cloud Infrastructure." Research Advances in Cloud Computing. Springer, Singapore, 2017. 327-350.

[11] Tiwari, Pradeep Kumar, and Sandeep Joshi. "An Empirical Result Analysis of Dynamic Weighted Live Migration Mechanism for Load Balancing in Cloud Computing." International Journal of Energy Optimization and Engineering (IJEOE) 6.4 (2017): 51-65.

[12] Madni, Syed Hamid Hussain, Muhammad Shafie Abd Latiff, and Yahaya Coulibaly. "Resource scheduling for infrastructure as a service (IaaS) in cloud computing: Challenges and opportunities." Journal of Network and Computer Applications 68 (2016): 173-200.

[13] Xu, Minxian, Wenhong Tian, and Rajkumar Buyya. "A survey on load balancing algorithms for virtual machines placement in cloud computing." Concurrency and Computation: Practice and Experience 29.12 (2017).

[14] Choudhary, A., Govil, M.C., Singh, G., Awasthi, L.K., Pilli, E.S. and Kapil, D., 2017. A critical survey of live virtual machine migration techniques. Journal of Cloud Computing, 6(1), p.23.

[15] Han, Yi, et al. "Using virtual machine allocation policies to defend against co-resident attacks in cloud computing." IEEE Transactions on Dependable and Secure Computing 14.1 (2017): 95-108.

[16] Xu, Shuting, et al. "Energy-Efficient Dynamic Consolidation of Virtual Machines in Big Data Centers." International Conference on Green, Pervasive, and Cloud Computing. Springer, Cham, 2017.

[17] Khosravi, Atefeh, Adel Nadjaran Toosi, and Rajkumar Buyya. "Online virtual machine migration for renewable energy usage maximization in geographically distributed cloud data centers." Concurrency and Computation: Practice and Experience (2017).

[18] Duggan, Martin, et al. "A network aware approach for the scheduling of virtual machine migration during peak loads." Cluster Computing (2017): 1-12.

[19] Wu, Tin-Yu, Nadra Guizani, and Jhih-Siang Huang. "Live migration improvements by related dirty memory prediction in cloud computing." Journal of Network and Computer Applications 90 (2017): 83-89.

[20] Sohrabi, Sahar, et al. "Energy-efficient adaptive virtual machine migration mechanism for private Clouds." Concurrency and Computation: Practice and Experience29.18 (2017).

[21] Teng, Fei, et al. "Energy efficiency of VM consolidation in IaaS clouds." The Journal of Supercomputing 73.2 (2017): 782-809

[22] Zola, Enrica, and Andreas J. Kassler. "Optimising for energy or robustness? Trade-offs for VM consolidation in virtualized datacenters under uncertainty." Optimization Letters 11.8 (2017): 1571-1592.

[23] Abdullah, Monir, et al. "A Heuristic-Based Approach for Dynamic VMs Consolidation in Cloud Data Centers." Arabian Journal for Science and Engineering (2017): 1-15.

[24] Kansal, Nidhi Jain, and Inderveer Chana. "Energy-aware virtual machine migration for cloud computing-a firefly optimization approach." Journal of Grid Computing 14.2 (2016): 327-345. 